\title{
Tuberculosis in Greece: bacteriologically confirmed cases and anti-tuberculosis drug resistance, 1995-2009
}

\author{
D Papaventsis (dpapaventsis@yahoo.gr) ${ }^{1}$, S Nikolaou ${ }^{1}$, S Karabela ${ }^{1}$, P Ioannidis $^{1}$, E Konstantinidou ${ }^{1}$, I Marinou ${ }^{1}$, A Sainti $^{1}$, \\ S Kanavaki ${ }^{1}$ \\ 1. National Reference Laboratory for Mycobacteria, Sotiria Chest Diseases Hospital, Athens, Greece
}

Citation style for this article:

Papaventsis D, Nikolaou S, Karabela S, loannidis P, Konstantinidou E, Marinou I, Sainti A, Kanavaki S. Tuberculosis in Greece: bacteriologically confirmed

cases and anti-tuberculosis drug resistance, 1995-2009. Euro Surveill. 2010;15(28):pii=19614. Available online: http://www.eurosurveillance.org/ViewArticle. aspx?Articleld $=19614$

Article published on 15 July 2010

The Greek National Reference Laboratory for Mycobacteria is a major source of tuberculosis (TB)related data for Greece, where the TB burden and epidemiology still need to be better defined. We present data regarding newly diagnosed TB cases and resistance to anti-TB drugs during the last 15 years in Greece. Although the total number of newly detected TB cases has declined, cases among immigrants are increasing. Resistance to first-line anti-TB drugs is widely prevalent, although stable or declining. The implementation of an efficient and effective countrywide TB surveillance system in Greece is urgently needed.

\section{Introduction}

Despite remarkable efforts to control tuberculosis (TB), the disease remains prevalent worldwide, and important issues regarding drug resistance have emerged $[1,2]$. In Greece, according to the recently published report by the European Centre for Disease prevention and Control and the World Health Organization (WHO) Regional Office for Europe, the case notification rate was 6.0 cases per 100,000 population in 2008 , while only $37.7 \%$ of all reported TB cases were confirmed by bacterial culture [3]. Underreporting is an important problem for TB control in Greece, where various limitations in the national TB monitoring system exist [4]. Reluctance to notify TB cases and failure to collect data at regional and national level makes TB surveillance and trend analysis problematic. Drug-resistant TB is common among repatriated Greeks from the former Soviet Union (FSU; principally Kazakhstan, Russia, Georgia, Uzbekistan, and Kyrgyzstan). Furthermore, migration from regions with high TB incidence (Iraq, Afghanistan, India, Africa etc.) possibly leads to further underestimation of the TB burden and facilitates further spread of the disease.

The Greek National Reference Laboratory for Mycobacteria (NRLM) provides a variety of reference services for both the public and the private healthcare sector in the field of mycobacterial disease. This laboratory constitutes a major source of TB-related data for Greece. Detection, identification and drug susceptibility testing (DST) for first-line anti-TB drugs, are routinely performed for every confirmed case by both conventional and molecular techniques. In addition, DST for second-line drugs is performed for multidrugresistant (MDR) TB cases.

This report presents the results of the analysis of all culture-confirmed newly diagnosed TB cases referred to the Greek NRLM between 1995 and 2009. A special focus of the report was to document the prevalence of Mycobacterium tuberculosis (MTB) resistance against the first-line anti-TB drugs among the native Greek population and immigrants/foreign-born, and especially isoniazid (INH) and rifampicin (RIF), due to their importance for a successful anti-TB treatment.

\section{Methods}

We studied 7,042 MTB strains. Only newly diagnosed cases (referred to as 'new cases' in the following) and one sample per patient were included. Patients were categorised as Greeks or immigrants, as declared by the patients themselves on the basis of nationality or, in the case of repatriated Greeks, place of birth. Repatriated Greeks were categorised for the purpose of his study, in the group of immigrants/foreign-born. No ethical approval was required for this study. ZiehlNeelsen staining, direct microscopy and culture in solid Löwenstein-Jensen medium (LJ) and liquid culture media using initially the Bactec 460 and since 2003 the Bactec MGIT 960 mycobacterial detection systems (Becton Dickinson, Sparks, US) were performed. Bacterial identification was based on conventional phenotypic tests and molecular characterisation using commercially available methods. For first-line drugs, DST was performed in solid LJ (using the proportion method) and MGIT 960 according to the manufacturer instructions. Since 2007, Genotype MTBDRplus (Hain Lifescience, Nehren, Germany) has also been used as a rapid molecular diagnostic tool for MDR-TB detection. For second-line drugs, DST of MDR-TB strains was performed using MGIT 960 during the last five years of the study. Drug concentrations used were according to the WHO guidelines [5]. 


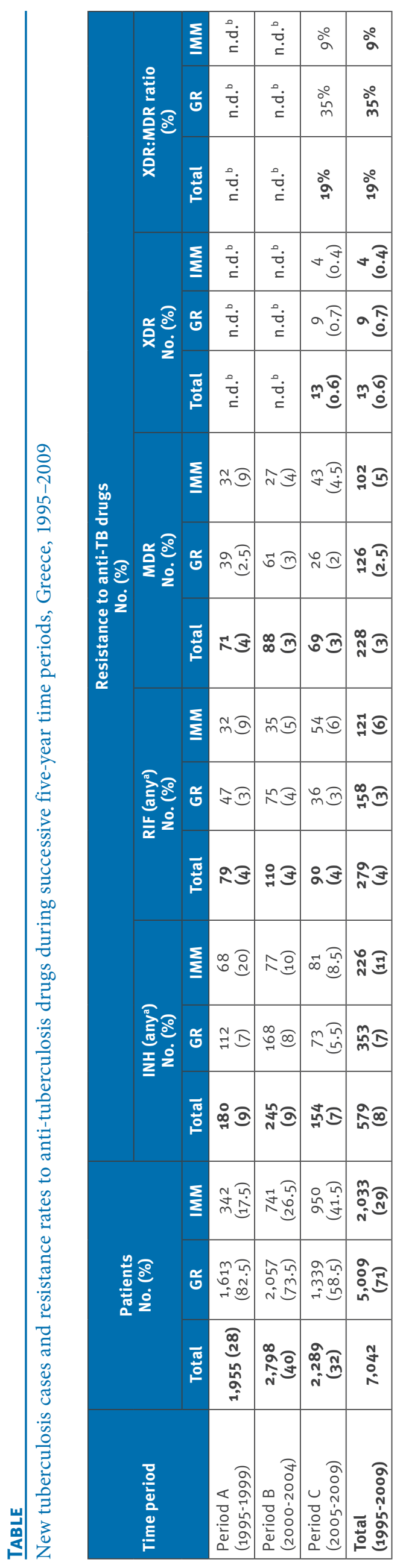

Differences in resistance rates were compared using the chi-square test. Statistical significance was set at $95 \%$. Odds ratios and coefficients of determination $\left(R^{2}=\right.$ the square of the sample coefficient) were also calculated. Statistical analyses were performed using GraphPad Prism software V.5 (GraphPad, San Diego, US).

\section{Results}

Among 7,042 new TB cases, 5,009 (71\%) were Greeks and 2,033 (29\%) were immigrants/foreign born (Table). The total number of new cases per year increased from 320 in 1995 to a peak of 650 in 2000 , and has since then steadily declined to 441 cases in 2009 (Figure 1). New cases among Greeks followed a similar trend. During the same period, the total number of new cases in immigrants increased almost sixfold from 40 cases (1995) to 240 (2009). In fact, 2009 was the first year when the absolute number of new cases among immigrants/foreign born was higher than among Greeks (240 versus 201 cases, respectively). New cases among immigrants accounted for $54.4 \%$ of total TB cases in 2009 (compared with $12.5 \%$ in 1995).

In total, 6,130 of the 7,042 TB isolates (87\%) were found sensitive to all anti-TB drugs; 912 isolates (13\%) were resistant to at least one drug (Figure 2). Drug resistance data for INH, RIF, MDR-TB and extensively drug-resistant (XDR)-TB during three successive time periods of five years (A: 1995-1999; B: 2000-2004; C: 2005-2009) are also presented in the Table. Resistance to INH decreased among Greeks ( $8 \%$ in period B to $5.5 \%$ in period $C$ ), a statistically significant difference $(p=0.0026)$. The same applies to the immigrants group, with a decrease from $20 \%$ in period $A$ to $8.5 \%$ in period C ( $p<0.0001)$. Resistance to RIF remained constant among Greeks during the whole time period $(p=0.7124)$, while it dropped from $9 \%($ period $A)$ to $6 \%$ (period C) among immigrants $(p=0.0195)$. Resistance to INH or RIF was more likely to be found in the immigrants group during the whole time period of the report. The MDR-TB rate $(2.5-3 \%)$ remained constant among Greeks $\left(R^{2}=0.0037 ; p=0.3802\right)$, while it decreased from $9 \%$ (period A) to $4.5 \%$ (period C) among immigrants $\left(R^{2}=0.1484 ; p=0.0011\right)$. MDR-TB was 2.04 more common in immigrants compared to Greeks (OR: 2.05; $95 \%$ Cl: 1.57-2.67) (Figure 3).

Mono-resistance to streptomycin (SM) was detected in 245 isolates ( $26 \%$ of resistance phenotypes; $3.5 \%$ of cases), and this was the most frequent resistance phenotype followed by mono-resistance to INH (148 isolates; $16 \%$ of phenotypes; $2 \%$ of cases), combined resistance to SM+INH (136 isolates; $15 \%$ of phenotypes; $1.9 \%$ of cases) and resistance phenotype $\mathrm{SM}+\mathrm{INH}+\mathrm{RIF}+$ Ethambutol (EMB) (84 isolates; $9 \%$ of phenotypes; $1.2 \%$ of cases). Combined resistance to all first-line anti-TB drugs was found in 26 isolates ( $3 \%$ of phenotypes; $0.36 \%$ of all cases). Mono-resistance to RIF was rather uncommon $(n=33 ; 4 \%$ of phenotypes; $0.46 \%$ of new cases), confirming a RIF mono-resistance 
rate of $0.38 \%$ previously reported [6]. Finally, since the year 2006, 13 XDR-TB cases (0.6\%) have been recorded in the Greek NRLM, nine (0.7\%) cases among Greeks and four $(0.4 \%)$ cases among immigrants $(p=0.8798)$.

\section{Discussion}

In the period under investigation, and according to the 2001 census, the population of Greece increased from $10,259,900$ in 1991 to $10,964,020$ in 2001 [6]. It is estimated that this increase is attributable almost entirely to immigration, with 762,191 'foreigners' living

\section{FIGURE 1}

Bacteriologically confirmed new tuberculosis cases per year, Greek National Reference Laboratory for Mycobacteria, 1995-2009

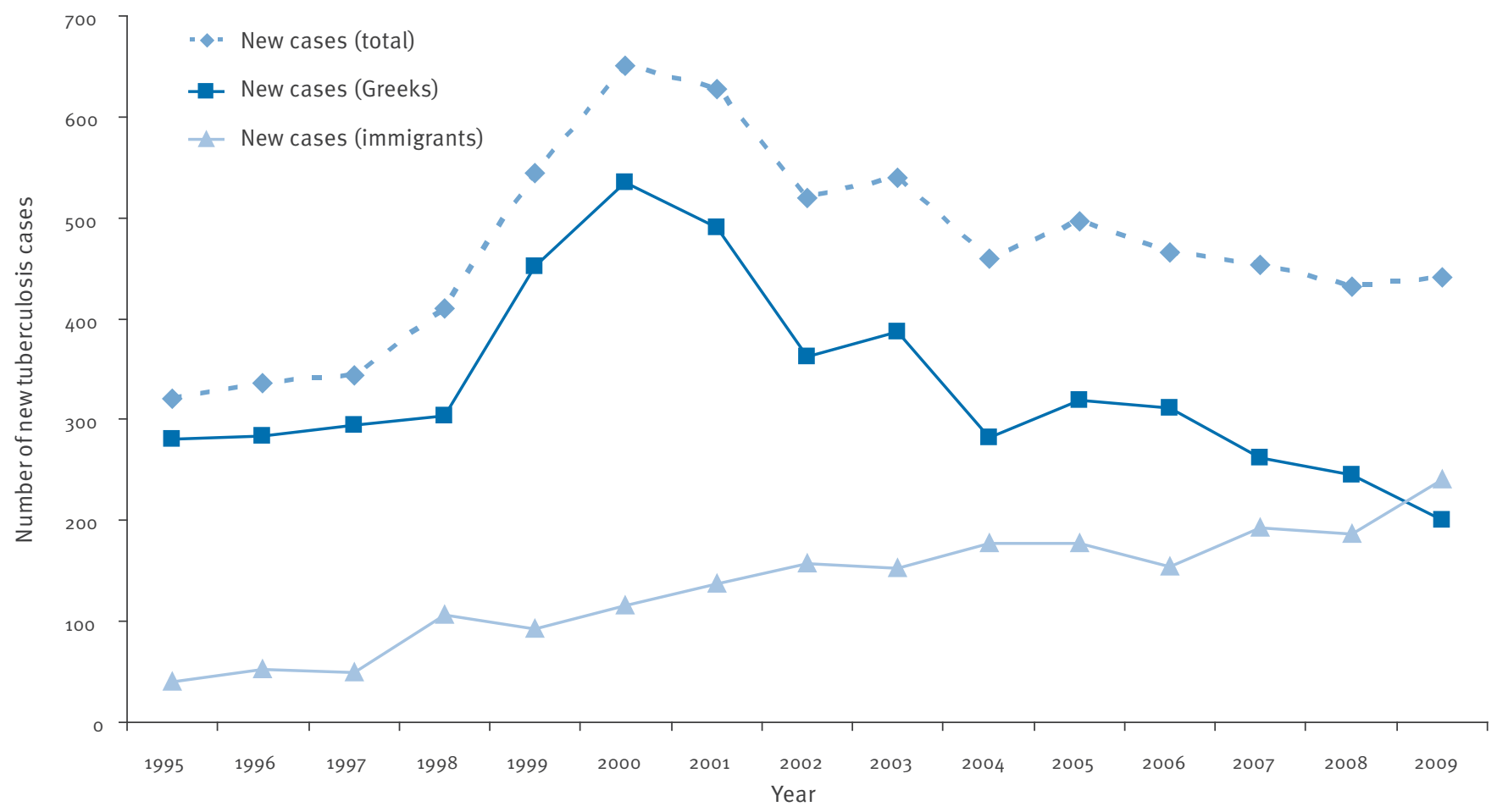

\section{FIGURE 2}

Drug susceptibility testing data and tuberculosis resistance phenotypes rates, Greece, 1995-2009

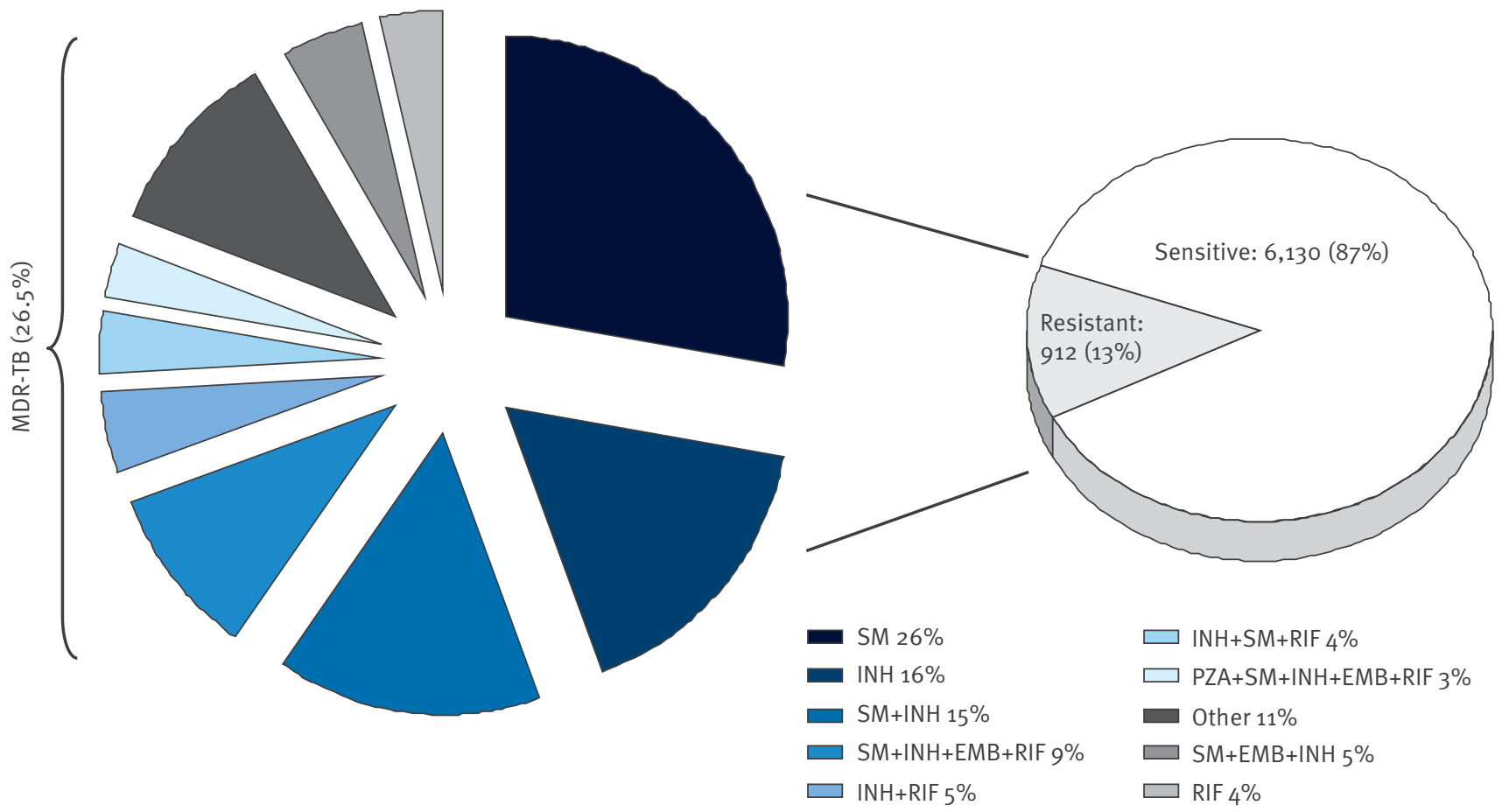

EMB: ethambutol; INH: isoniazid; MDR: multidrug-resistant; PZA: pyrazinamide; RIF: rifampicin; SM: streptomycin; TB: tuberculosis. 
in Greece in 2001, approximately $7 \%$ of total population [6]. Greece has received several hundred thousands of immigrants from countries with a higher TB prevalence. In 1998, the implementation of countrywide immigrants' health inspection programmes contributed to the better TB management. After a sharp increase in TB incidence in 2000 , possibly connected to a peak of immigration from the FSU (including repatriated Greeks) and to better case reporting, new TB cases per year in total and among Greeks decreased. As documented by this report and also by Kanavaki et al. [7], the number of new TB cases recorded among immigrants has increased sixfold during the past 15 years. A similar trend has previously been reported for pooled data from other European countries [8]. In line with this trend, the present study found that in 2009, the absolute number of new cases among immigrants/foreign born was for the first time higher than among Greeks. This trend could be attributed to several factors, including improved accessibility to healthcare services for immigrants. However, the degree of undernotification of TB cases remains high, leading to substantial underestimation of the disease burden in Greece [4].

Another important finding of the study was the decreasing prevalence of MTB resistant to INH in both study groups. From period $A$ to period $C$, the prevalence of RIF-resistant and MDR-TB strains decreased by almost half among immigrants, which could be attributed to the fact that immigration to Greece from the FSU states, where RIF resistance and MDR-TB was highly prevalent, culminated in the early 1990s [6]. However, previous [7] and current data confirm that the absolute number of resistant cases in immigrants still appears to be increasing.

Resistance to first-line anti-TB drugs remains higher in Greece than in most other countries in western Europe $[9,10]$ : Resistance to INH was slightly higher in Norway (10.4\%), Sweden (9.9\%), Austria (9.5\%) and Luxemburg (8.3\%). Much higher INH resistance rates were observed in FSU countries, for example: Kazakhstan (42.6\%), Latvia (30.9\%), Russia (26\%), Georgia (23.4\%), Estonia (20.6\%), and Lithuania (20.3\%). RIF resistance was also higher in Greece ( $4 \%$ ) compared with other western European countries. MDR-TB was much higher in Kazakhstan (14.2\%), Estonia (13.3\%), Russia (12.5\%), Latvia (10.8\%), Lithuania (9.8\%) and Georgia (7.6\%) than in Greece ( $4 \%$ ) and the rest of Europe (median European MDR rate $1.0 \%$ ) [10]. Knowledge of the epidemiology of resistance patterns in a country is critical for the introduction of national guidelines for the management of tuberculosis. Streptomycin monoresistance appears to be very common in Greece. Data regarding second-line drugs are limited and should be interpreted with caution.

The current report has several limitations. Firstly, the representativeness of the sample is unknown. Sampling bias, especially among immigrants/foreign born, could not be excluded. Although access of the immigrant population to the national healthcare system has improved over the years, many patients among

\section{FIGURE 3}

Percentage of multidrug resistance, Greeks versus immigrants/foreign-born, Greece, 1995-2009

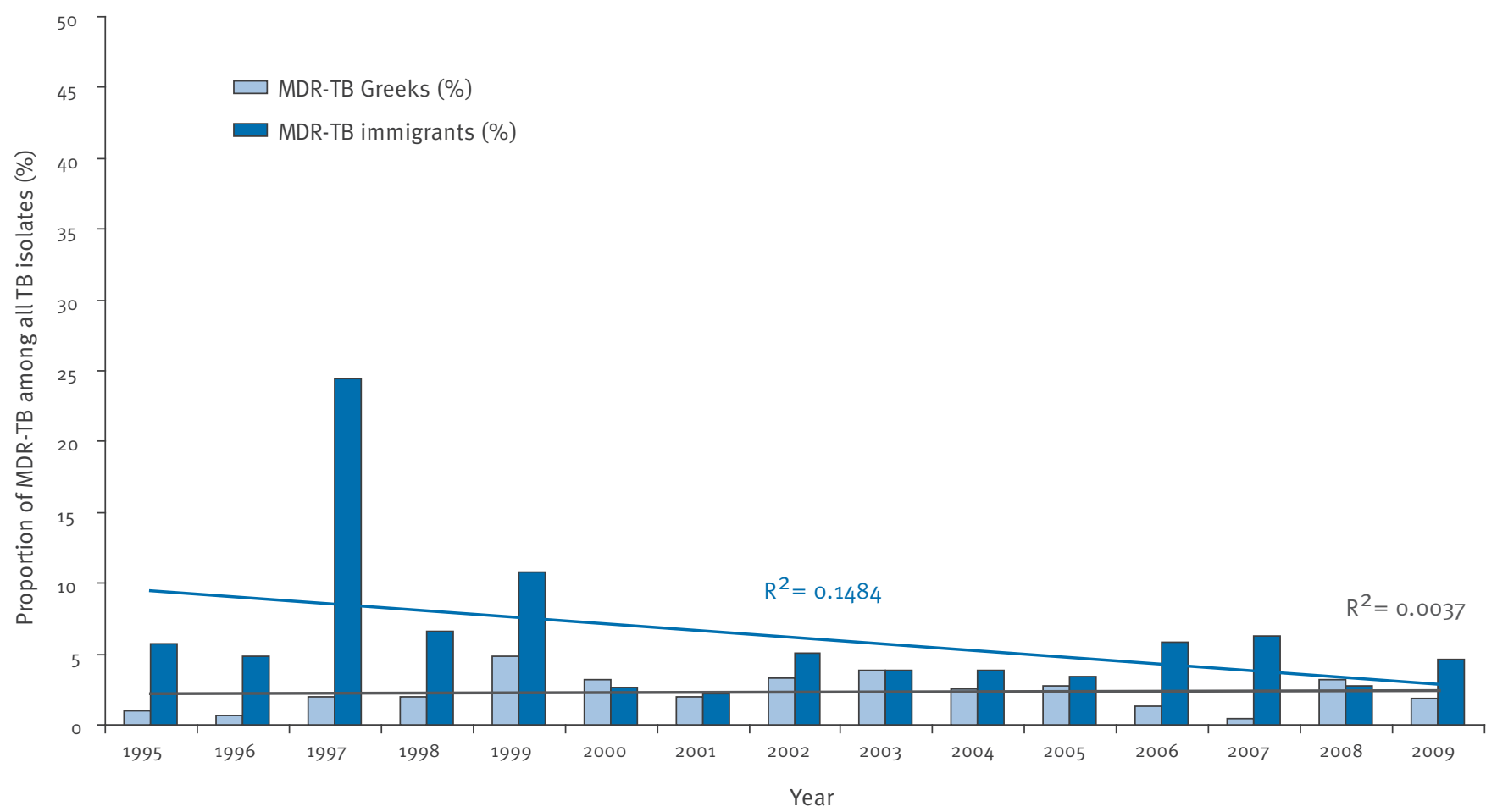


foreigners living in Greece do not have full access to hospital services. In addition, repatriated Greeks from the FSU could not always be clearly identified within the native Greek population. Secondly, incomplete patient records, lacking data on epidemiological risk factors (such as belonging to a vulnerable group, previous treatment status, sex and age), and the lack of genotyping data for MTB isolates, limited the estimation of resistance transmission among the study population. The implementation of a nationwide TB Genotyping and Surveillance Network will be essential for tuberculosis control in Greece.

\section{Acknowledgements}

The authors would like to thank technicians Spyridoula Anagnostou, Paraskevi Karfi, Maria Kolettou, Kyriaki Toumbaniari, Ekaterini Raftopoulou, Marina Panagi, and Anastasios Skouroglou for their technical assistance and also all colleagues who worked in the lab during the last 15 years.

Updated data regarding new TB cases and anti-TB drugs resistance in the NRLM can be accessed via the Greek System for the Surveillance of Antimicrobial Resistance (WHONET GREECE) webpage at http://www.mednet.gr/whonet/. We would like to thank Professor Alkiviadis Vatopoulos and $\mathrm{Mr}$ Michael Polemis for their support.

This report was funded internally by the Sotiria Chest Diseases Hospital, Athens, Greece.
10. Wright A, Zignol M, Van Deun A, Falzon D, Gerdes SR, Feldman $\mathrm{K}$, et al. Epidemiology of antituberculosis drug resistance 2002-07: an updated analysis of the Global Project on AntiTuberculosis Drug Resistance Surveillance. Lancet. 2009; 373 (9678):1861-73.

\section{References}

1. World Health Organization (WHO). Global Tuberculosis Control. A short update to the 2009 report. Geneva; WHO; 2009. Available from: http://www.who.int/tb/publications/global_ report/2009/update/tbu_9.pdf

2. World Health Organization (WHO). Global Tuberculosis Control 2009: epidemiology, strategy, financing. Geneva: WHO; 2009. Available from: http://www.who.int/tb/publications/global_ report/2009/pdf/full_report.pdf

3. European Centre for Disease Prevention and Control (ECDC)/ WHO Regional Office for Europe. Tuberculosis surveillance in Europe 2008. Stockholm: ECDC; 2010. Available from: http:// www.ecdc.europa.eu/en/publications/Publications/1003_SUR tuberculosis surveillance in europe 2008.pdf

4. Jelastopulu E, Alexopoulos EC, Venieri D, Tsiros G, Komninou G, Constantinidis TC, et al. Substantial underreporting of tuberculosis in West Greece - implications for local and national surveillance. Euro Surveill. 2009;14(11):pii=19152. Available from: http://www.eurosurveillance.org/ViewArticle. aspx?Articleld $=19152$

5. World Health Organization (WHO). Policy guidance on drugsusceptibility testing (DST) of second-line anti-tuberculosis drugs. Geneva: WHO; 2008. Available from: http://www.who. int/tb/publications/2008/who_htm_tb_2008_392.pdf .

6. Migration Policy Institute (MPI). [Internet]. Greece: A History of Migration. Washington DC: MPI. [Accessed March 2010]. Available from: http://www.migrationinformation.org/Profiles/ display.cfm?ID=228

7. Kanavaki S, Mantadakis E, Nikolaou S, Papavassiliou A, Karambela S, Anagnostou S, et al. Resistance of Mycobacterium tuberculosis isolates in different populations in Greece during 1993-2002. Int J Tuberc Lung Dis. 2006; 10(5):559-64.

8. Falzon D, Kudjawu Y, Desenclos JC, Fernandez de la Hoz K, Dadu A, et al. Stopping TB in Europe: some progress but still not there. Euro Surveill. 2008;13(12): pii=8073. Available from: http://www.eurosurveillance.org/ViewArticle. aspx?Articleld $=8073$

9. World Health Organization (WHO). Anti-Tuberculosis Drug Resistance in the World. Report No.4.The WHO/IUATLD Global Project on Anti-Tuberculosis Drug Resistance Surveillance 2002-2007. Geneva: WHO; 2008. Available from: http://www. who.int/tb/publications/2008/drs report4_26febo8.pdf 